\section{Exploiting natural killer group 2D receptors for CAR T-cell therapy}

\author{
Benjamin Demoulin', W. James Cook², Joana Murad ${ }^{\ddagger, 3}$, David J Graber, ${ }^{\ddagger}$, \\ Marie-Louise Sentman ${ }^{\ddagger 2}$, Caroline Lonez', David E Gilham', \\ Charles L Sentman² \& Sophie Agaugue ${ }^{*, 1}$
}

Chimeric antigen receptors (CARs) are genetically engineered proteins that combine an extracellular antigen-specific recognition domain with one or several intracellular T-cell signaling domains. When expressed in T cells, these CARs specifically trigger T-cell activation upon antigen recognition. While the clinical proof of principle of CAR T-cell therapy has been established in hematological cancers, CART cells are only at the early stages of being explored to tackle solid cancers. This special report discusses the concept of exploiting natural killer cell receptors as an approach that could broaden the specificity of CART cells and potentially enhance the efficacy of this therapy against solid tumors. New data demonstrating feasibility of this approach in humans and supporting the ongoing clinical trial are also presented.

First draft submitted: 6 March 2017; Accepted for publication: 19 April 2017; Published online: 14 June 2017

\section{Cancer immunotherapy}

While surgery, chemotherapy and radiation remain the mainstays of current cancer therapy, exploiting the power of the immune system to target tumors (cancer immunotherapy) is emerging as a new paradigm to complement standard therapy. Engineering $T$ cells by means of a chimeric antigen receptor (CAR) has recently provided highly encouraging results in patients with certain hematological cancers. CAR T cells are genetically engineered $\mathrm{T}$ cells displaying, at their cell surface, artificial receptors that recognize tumors expressing specific antigen $(s)[1,2]$. The targeting domain of this artificial receptor is generally coupled to an intracellular T-cell stimulatory domain (CD3乙) and depending on the CAR generation, one or two intracellular costimulatory domains (e.g., CD28 and CD137). Engagement of the extracellular domain of the CAR with its corresponding ligand triggers antigen-specific T-cell activation resulting in cytokine release and tumor cell killing. Most of the clinical data currently available describes CD19-specific CAR T-cell therapy for patients with B-cell malignancies, where many objective responses in early phase clinical trials have been observed. However, to date, CAR T-cell therapy in solid tumors has failed to achieve similar levels of clinical success [3-5]. There are many reasons that can explain poor efficacy in solid tumors, such as heterogeneous expression of the target, immunosuppressive microenvironment, lack or difficulty of access to the tumor, and poor trafficking of CAR T cells to the tumor site. In addition, the antibody-derived single chain antibody fragments (single chain fragment variable), typically used in classical CAR

'Research \& Development Department, Celyad SA, Mont-Saint-Guibert, Belgium

${ }^{2}$ Center for Synthetic Immunity, Department of Microbiology \& Immunology, Geisel School of Medicine, Dartmouth College, Lebanon, $\mathrm{NH}$, USA

${ }^{3}$ Celdara Medical, LLC, Lebanon, NH, USA

*Author for correspondence: sagaugue@celyad.com

¥Authors contributed equally

\section{KEYWORDS}

- adoptive cell therapy

- CAR-T • chimeric antigen

receptor $\bullet$ clinical trial

- CM-CS1 • immunotherapy

- NKG2D • NKR-2

- pancreatic cancer 
constructs only recognize one antigen. On one hand a single chain fragment variable provides a high specificity, but on the other hand, in a situation where the CAR $\mathrm{T}$ cell is not able to easily access its target, where the target is missing due to tumor heterogeneity or antigen escape mechanisms, effective therapeutic activity is challenging. As an alternative to these classical CAR limitations and to provide more options to target solid tumors, efforts are underway to exploit 'natural' receptors recognizing multiple antigens for CAR T-cell therapy.

\section{- Natural killer group 2D protein to target T cells to solid \& hematological tumors} Natural killer $(\mathrm{NK})$ cells are part of the innate immune system and are naturally capable of killing tumor cells and virally infected cells. In addition, NK cells secrete cytokines that assist in establishing an adaptive immune T-cell response and recruiting other immune cells, predominantly via interferon (IFN) $-\gamma$ and tumor necrosis factor (TNF)-a secretion [6]. Their effector functions are tightly controlled by a balance between activating and inhibitory signals mediated by receptors at their surface [7].

To date, a well-characterized NK activating receptor is the NKG2D receptor, a C-type lectin-like, type II transmembrane glycoprotein. In humans, NKG2D is expressed on NK cells, NK $\mathrm{T}$ cells, $\mathrm{CD}^{+} \mathrm{T}$ cells and, under some conditions, in a subset of $\gamma^{+}$and $\mathrm{CD}^{+} \mathrm{T}$ cells $[8,9]$. To achieve expression at the cell surface, NKG2D associates with an adaptor molecule called DNAX-activating protein of $10 \mathrm{kDa}$ (DAP10). The cytoplasmic domain of DAP10 actively participates in cell activation since it is phosphorylated upon interaction of NKG2D with its

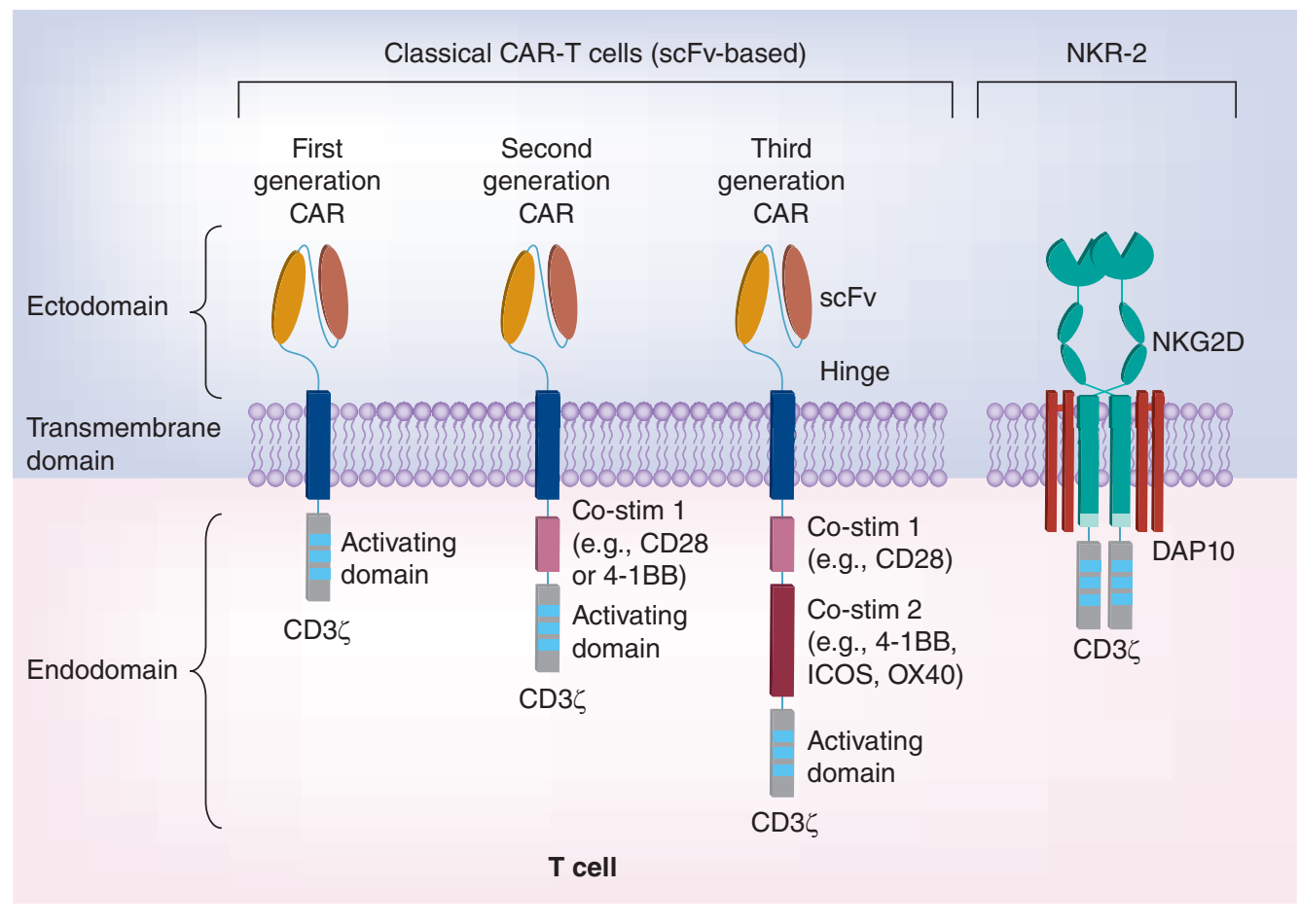

Figure 1. Comparison between classical chimeric antigen receptor and NKR-2. Classical CAR T cells are composed by an antigen-specific antibody-like ectodomain (single chain fragment variable) fused to a hinge, a transmembrane domain, an intracellular activating domain (CD3ろ) and, depending on the CAR generation, to one or two costimulatory domains. NKR-2 uses the NKG2D complex to provide primary and costimulation signals: the full length NKG2D protein (ectodomain, transmembrane and intracellular domains) is fused with an inverted intracellular domain of CD3 . Moreover, DAP10, that stabilizes the complex, also triggers a costimulatory signal that makes NKR-2 a second-generation CAR. By contrast to a classical CAR specific for one target, the NKG2D ectodomain recognizes multiple ligands and allows the potential recognition of many tumor types.

CAR: Chimeric antigen receptor. 


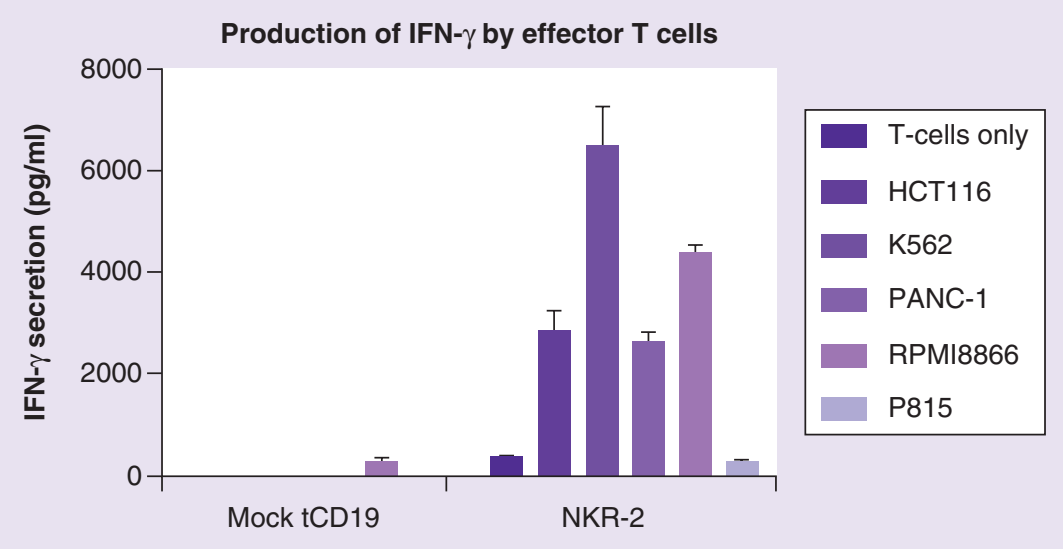

Figure 2. NKR-2 T cells secrete IFN- $\gamma$ when cultured in presence of NKG2D ligand-expressing cancer cell lines. Human T cells retrovirally transduced with the truncated form of CD19 (tCD19) or with the NKR-2 construct containing the tCD19 tag were incubated for 24 h, 1:1 E:T ratio, with cancer cell lines expressing NKG2DL: HCT-116 (human colon tumor cell line); K562 (human chronic myelogenous leukemia); PANC-1 (human pancreatic tumor cell line) and RPMI8866 (human leukemic cell line). A NKG2DL-negative cancer cell line, P815 (a murine mastocytoma cell line) was also included. After $24 \mathrm{~h}$ of incubation, cell-free medium was harvested and tested for IFN- $\gamma$ by ELISA. Mock tCD19 T cells were not activated while incubated with cancer cell lines. On the contrary, NKR-2 transduced T cells secreted large amounts of IFN- $\gamma$ when incubated with NKG2DL-positive cell lines but not with P815 cells indicating that NKR-2 T cells require NKG2DL expression on tumor cell lines. Results are the mean \pm SD of triplicates, and data are representative of four to six experiments.

ligand. Then DAP10 recruits and activates the p85 subunit of phosphoinositide-3-kinase and the growth factor receptor-bound protein $2 /$ vav guanine nucleotide exchange factor 1 complex, which triggers downstream signaling cascades leading to effector activities [10,11].

In humans, two families of NKG2D ligands (NKG2DL) have been identified, the MHC class I chain related proteins A (MICA) and $\mathrm{B}$ (MICB) and the structurally diverse unique long 16 binding proteins, in other words, UL16binding proteins 1 to 6 (ULBP1-6). NKG2DL expression is tightly regulated by transcriptional, translational and post-translational mechanisms in healthy adult tissues to prevent self-recognition and autoimmune reactivity [12]. However, cell surface protein expression may be induced by various stress situations, such as viral infection, oxidative or thermal stress, genotoxic drugs, tissue damage, heat shock, inflammation (e.g., autoimmune diseases) or malignant transformation [12,13]. Accordingly, many tumor cell lines and primary tumors from diverse tissue origins express NKG2DL (see Table 1) implying NKG2DL to be putative targets on both solid and hematological tumors $[14,15]$.

\section{NKG2D CAR T cells}

In 2005, Professor Charles Sentman's team tested the fusion of the full length NKG2D protein with $\mathrm{CD} 3 \zeta$ as a CAR expressed in primary T cells [16]. At first sight, this NKG2D-CD3ろ fusion construction is built as a first-generation CAR (Figure 1). However, as mentioned earlier, NKG2D's natural binding partner DAP10 also triggers a costimulatory signal while NKG2D recognizes its ligands [17-19]. Thus, the NKG2DCD3 CAR initiates both activation signal 1 and signal 2 upon ligand binding in a manner akin to a second-generation CAR. With this design, the NKG2D-CD3ろ CAR takes advantage of the configuration of the NKG2D receptor complex with two NKG2D molecules and four DAP10 molecules.

\section{- Preclinical development}

Over the last decade, the Sentman laboratory has carried out an extensive analysis of the in vitro functional activity of $\mathrm{T}$ cells expressing the NKG2D-CD3ろ CAR (hereafter termed chimeric NKG2D [chNKG2D] when it is referring to the murine NKG2D CAR, and NKR-2 when it is the human NKG2D CAR) on cancer cell lines as 
well as in vivo experiments in mouse tumor models. Preclinical data generated with chNKG2D $T$ cells derived from murine cells demonstrate the huge potential of this technology to target solid and hematologic tumor types in immunocompetent syngeneic murine models of ovarian cancer [20], melanoma [21], lymphoma [22] and multiple myeloma (MM) [23]. In addition to this previous work, we report here new data demonstrating, for the first time, that human NKR-2 $\mathrm{T}$ cells are effective in vitro and in vivo in a mouse xenograft model of human pancreatic cancer.
As shown in Figure 2, in vitro coculture of human NKR-2 T cells with human cancer cell lines from multiple origins provided valuable information. First, NKR-2 T cells released IFN- $\gamma$ upon coculture with a colorectal cancer cell line (HCT-116), a pancreatic cancer cell line (PANC-1) and leukemia cell lines (K562 and RPMI8866) all expressing NKG2DL, therefore confirming the work previously done $[24,25]$ and extending the range of cancers potentially treatable with NKR-2. Second, NKR-2 T cells were not activated by cells not expressing human

(A)

NKR-2 potency:PANC-1
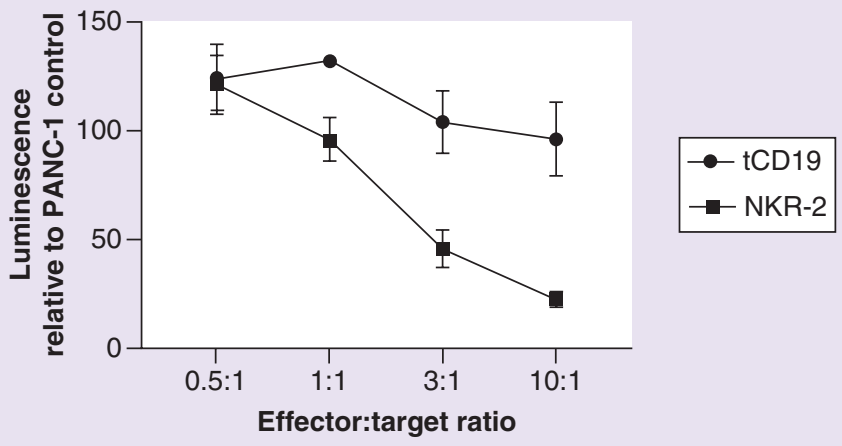

(B)

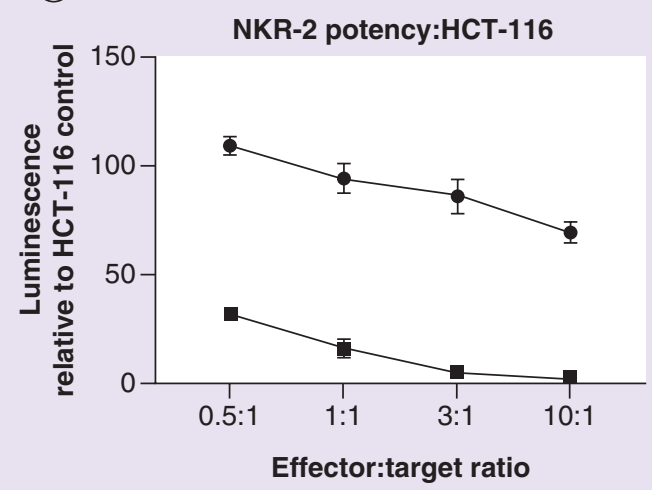

(C)

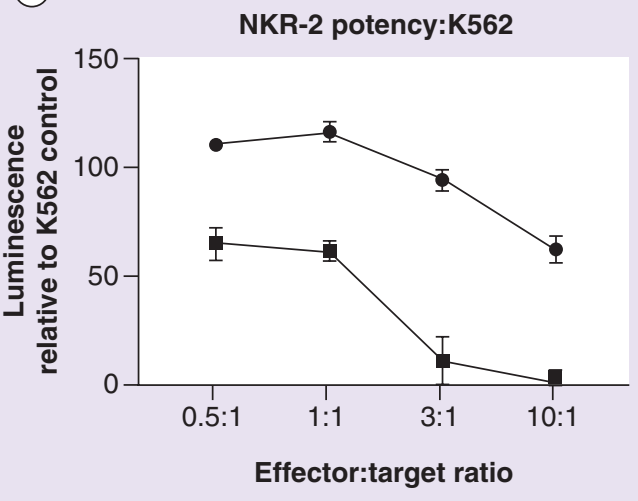

Figure 3. NKR-2 T cells display cytotoxic activity against NKG2D ligand-positive cancer cell lines. PANC-1, HCT-116 and K562 stably expressing luciferase were cocultured at various E:T ratios with human T cells retrovirally transduced with tCD19 or with NKR-2. After $24 \mathrm{~h}$, the bioluminescent signal corresponding to remaining viable tumor cells was quantified. (A) Co-incubation of luciferase expressing PANC-1 cells with NKR-2 T cells led to a loss of tumor cells as the E:T ratio increased. At 10:1 ratio, only $23 \%$ of PANC-1 survived to NKR-2 cytolytic activity while $96.1 \%$ of cells were still alive in the mock condition. (B) Co-incubation of luciferase expressing HCT-116 cells with NKR-2 T cells led to a decrease in luminescence signal even at the lowest ratio $(0.5: 1)$ where $68 \%$ of HCT-116 cells were already eliminated. (C) Co-incubation of luciferase expressing K562 cells with NKR-2 T cells led to a similar decrease in luminescence signal even at the lowest E:T ratio (-35\%). Tumor cells alone $=100 \%$ luminescence. Results represent mean \pm SD of triplicates, and they are representative of two or three experiments. 


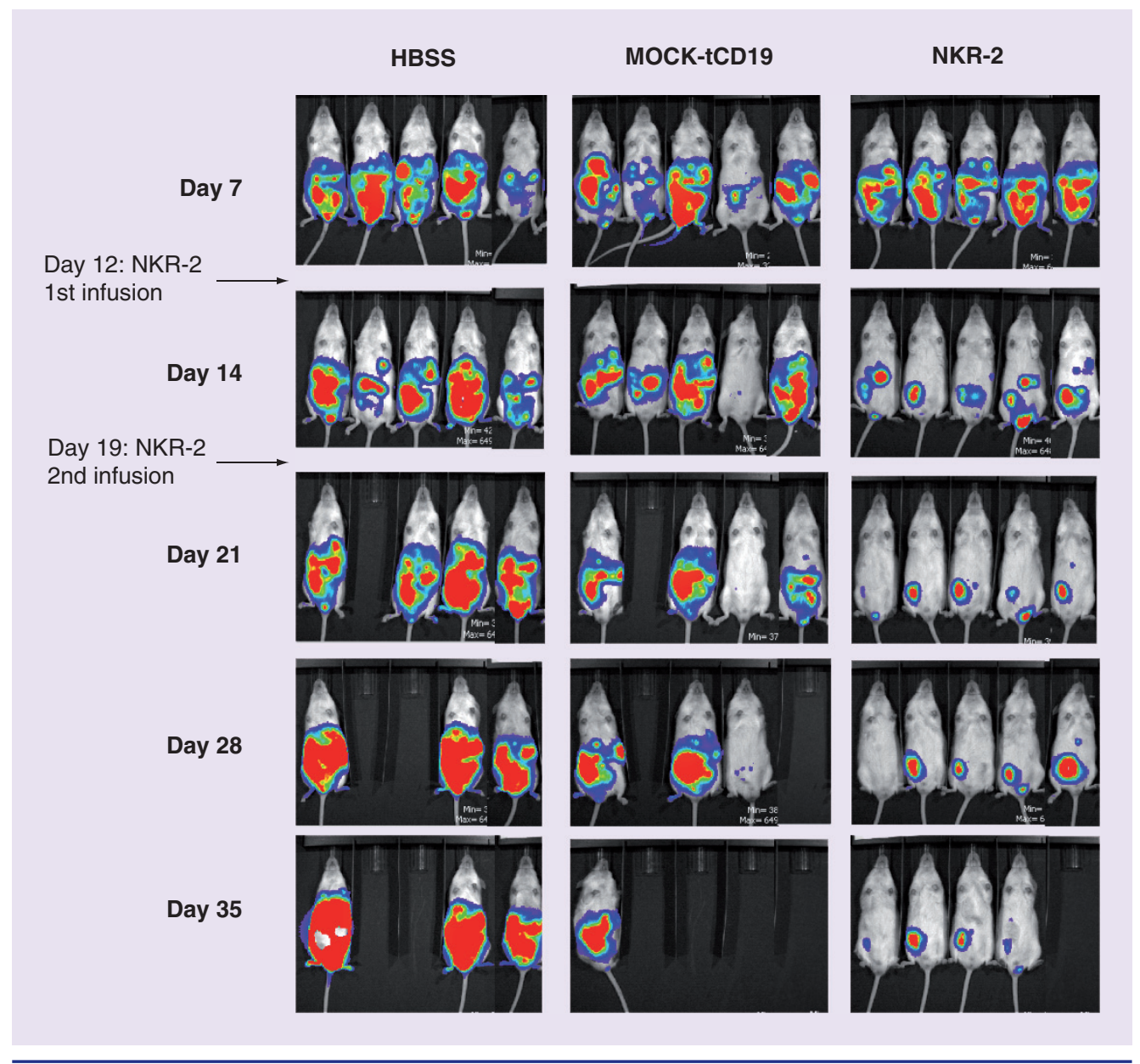

Figure 4. NKR-2 T cells inhibit PANC-1 progression in NOD-scid IL2Rgammanull mice. NSG mice were injected IP with $0.5 \times 10^{6}$ luciferase-expressing PANC-1 tumor cells and tumor engraftment was followed by bioluminescent imaging $(n=1)$. Mice in all treatment groups, except for mouse number 4 from mock tCD19 group (for which tumor injection failed), showed strong luminescence signal from the PANC-1 cells in the abdominal cavity at the first imaging prior to T cell injection (day 7), showing significant tumor burden. Twelve and nineteen days after PANC-1 injection, control mice received either HBSS (HBSS group), $5 \times 10^{6}$ of T cells (IP) retrovirally transduced with the tCD19 mock construct (mock-tCD19 group) or with the NKR-2 construct (NKR-2 group). Imaging was performed every week starting one week after tumor inoculation. The tumor cell luminescence was noticeably decreased and more localized in mice that received the NKR-2 T cells. Thirty-five days post tumor injection, the final imaging showed that some mice that received the NKR-2 treatment had little tumor remaining compared with HBSS and mock T cell groups where several mice had to be euthanized and the others had a large tumor burden. The in vivo efficacy of NKR-2 cells against PANC-1 cells was further confirmed in NSG mice IP injected with luminescent PANC-1 and IV treated with NKR-2 ( $n=2$, data not shown).

IP: Intraperitoneally; IV: Intravenously; NSG: NOD-scid IL2Rgamma ${ }^{\text {null. }}$

NKG2DL (the murine mastocytoma cell line P815), consistent with NKR-2 T cells secreting IFN- $\gamma$ when the target cells express NKG2DL hence decreasing the risk of potential 'off-target, off-tumor' toxicity issues.

The potential of the NKR-2 technology was further confirmed by demonstrating the in vitro cytolytic activity of NKR-2 T cells towards human NKG2DL-expressing cancer cell lines (Figure 3). Several previous studies showed that NKR-2 T cell lysis of tumor cells is dependent of NKG2D and NKG2DL [24,26]. Luciferaseexpressing tumor cell lines (PANC-1, HCT-116 and K562 cells) were co-incubated $24 \mathrm{~h}$ with 
NKR-2 T cells at different Effector:Target (E:T) ratios. A decrease of the bioluminescence signal was indicative of fewer tumor cells thus indicating specific killing of these tumor cells by NKR-2 T cells. As shown in Figure 3, the coculture of luciferase-expressing tumor cell lines with NKR-2 T cells led to a decrease in bioluminescence signal in a manner dependent of the E:T ratio compared with mock $\mathrm{T}$ cells (transduced with a retroviral vector only coding for the truncated form of human CD19). At the highest E:T ratio, the mock $\mathrm{T}$ cell cocultures showed some decreased luciferase signal, however NKR-2 T cell cocultures displayed a much lower level. Collectively, these results confirm that human NKR-2 has the potential to target multiple cancer indications.

The in vivo antitumor efficacy of human NKR-2 T cells in a xenograft model of human pancreatic cancer in NOD-scid IL2Rgamma ${ }^{\text {null }}$
(NSG) immunodeficient mice was then performed. As shown in Figure 4, intraperitoneal (IP) treatment of established PANC-1 tumors with human NKR-2 $\mathrm{T}$ cells led to a strong reduction of PANC-1 tumor growth. This diminution of the tumor mass was not observable in mice treated with mock T cells or HBSS (vehicle control). Moreover, additional experiments where NKR-2 T cells were intravenously (IV) injected showed decreased tumor growth compared with mock T-cell controls (data not shown). This suggests an efficient trafficking of NKR-2 T cells to the tumor site in this model. These results, in addition to those already published [20-25], provide a strong rationale that NKR-2 T cells can effectively target a range of established tumors, and can even lead to complete tumor eradication in many situations. This particularly supports the development of a clinical trial in pancreatic cancer. Moreover,

Table 1. NKG2D ligands expression on various cancer.

\begin{tabular}{|c|c|c|}
\hline Tumor type & Expressed NKG2D ligand & Ref. \\
\hline Acute lymphoblastic leukemia & $\begin{array}{l}28-67 \% \text { MICA/B } \\
9-20 \% \text { ULBP1-3 }\end{array}$ & {$[45-51]$} \\
\hline Acute myeloid leukemia & $\begin{array}{l}0-75 \% \text { MICA/B } \\
16-50 \% \text { ULBP1 } \\
4-64 \% \text { ULBP2 } \\
16-100 \% \text { ULBP3 }\end{array}$ & {$[45,46,49,50,52-62]$} \\
\hline Bladder carcinoma & $70 \% \mathrm{MICA}$ & {$[63,64]$} \\
\hline Brain cancer & $90 \%$ MICA, MICB and ULBP1-3 & {$[65-67]$} \\
\hline Breast cancer & $35-100 \%$ MICA/B, ULBP 1-5 & {$[68-72]$} \\
\hline Cervical cancer & $20 \%$ MICA, ULBP2 & {$[73,74]$} \\
\hline Chronic lymphocytic leukemia & $\begin{array}{l}0-85 \% \mathrm{MICA} / \mathrm{B} \\
10-20 \% \text { ULBP1-3 }\end{array}$ & {$[46,49,50,75,76]$} \\
\hline Chronic myeloid leukemia & $\begin{array}{l}28-100 \% \text { MICA/B } \\
12-20 \% \text { ULBP1-3 }\end{array}$ & {$[46,50,77,78]$} \\
\hline Colorectal cancer & $\begin{array}{l}80-100 \% \mathrm{MICA} / \mathrm{B} \\
\text { ULBP } 1-5\end{array}$ & {$[68,79,80]$} \\
\hline Gastric carcinoma & 40-100\% MICA/B, ULBP2 & [81-83] \\
\hline Hepatocellular carcinoma & $60-100 \%$ MICA & {$[84-87]$} \\
\hline Lymphoma & $\begin{array}{l}28-44 \% \text { MICA/B } \\
12-20 \% \text { ULBP1-3 }\end{array}$ & {$[48,50,51,88-95]$} \\
\hline Melanoma & $50 \% \mathrm{MICA} / \mathrm{B}$ & [96-99] \\
\hline Multiple myeloma & $\begin{array}{l}10-60 \% \text { MICA } \\
0-34 \% \text { ULBP1-3 }\end{array}$ & {$[36,100-105]$} \\
\hline Neuroblastoma & $86 \%$ MICA/B, ULBP1-3 & {$[106]$} \\
\hline Non-small-cell lung carcinoma & 20-30\% MICA/B, ULBP1-3 & {$[68,107-109]$} \\
\hline Ovarian carcinoma & 50-97\% MICA/B, ULBP1-5 & {$[68,110-113]$} \\
\hline Pancreatic cancer & $68-89.3 \% \mathrm{MICA} / \mathrm{B}$ & [114-116] \\
\hline Prostate cancer & 75-95\% MICA/B, sMICA/B & {$[68,117]$} \\
\hline Renal cell carcinoma & $>95 \% \mathrm{MICA} / \mathrm{B}$ & {$[68,78,118,119]$} \\
\hline Sarcoma & $100 \%$ MICA/B, ULBP 1-3 & {$[120,121]$} \\
\hline
\end{tabular}


as shown in syngeneic tumor models, animals that have eliminated tumors can resist to rechallenge with the same tumor cells without additional therapy [20]. Since no murine chNKG2D $\mathrm{T}$ cells were detected prior to rechallenge and host lymphocytes were able to now recognize the tumor, these findings indicate that the initial chNKG2D therapy induced a long-term memory immune response against unknown tumor antigen(s) by activating the immune system of the host.

In addition, and by contrast to a classical CAR, chNKG2D efficacy is independent of lymphodepleting preconditioning [23]. Moreover, chNKG2D was shown to be the most effective when cells were administered multiple times. Indeed, a single chNKG2D dose was shown to double the overall survival of treated mice compared with control; nevertheless, three doses were necessary to reach complete survival in a lymphoma model [22]. Interestingly, chNKG2D murine $\mathrm{T}$ cells also demonstrated antitumor efficacy on ovarian tumors composed of a mix of NKG2DLnegative and -positive tumor cells. chNKG2D $\mathrm{T}$ cells were still able to reduce ovarian tumor progression in tumors having $93 \%$ of NKG2DL-negative cells [27]. This strongly suggests that NKR-2 cells may be effective even in highly heterogeneous tumors. Together, this makes the NKR-2 approach very different than anti-CD19 CAR T cell therapy where a combination of a preconditioning chemotherapy with single dose of CAR T cells given shortly afterward remains essential for the success of the therapy [28].

Beyond this direct antitumor activity, murine models showed a change in the tumor microenvironment of animals receiving chNKG2D T cells. Indeed, while the immunosuppressive immunosuppressive $\mathrm{T}$ regulatory cells (Tregs) and myeloid-derived suppressor cells (MDSC) usually impair the cytotoxic ability of NK and T cells, it was shown that tumor-derived MDSCs [29] or Tregs [30,31] express NKG2DL and may be targeted by NKG2D-specific effector cells. Thus, NKR-2 putatively triggers a shift towards a proinflammatory environment and amplifies the natural immune system response to eradicate tumors.

Interestingly, there was also evidence of expression of NKG2DL on endothelial cells found in tumor neovasculature that supported observations of antitumor response in tumors that lack the expression of NKG2DL on their tumor cells [21].

Finally, as a potential mechanism of tumor immune escape, NKG2DL can be shed from the membrane of tumors cells and can even be measured in sera of cancer patients $(0-15 \mathrm{ng} / \mathrm{ml})$ [32]. The impact of these soluble NKG2DL (sNKG2DL) in the context of NKR-2 is still unknown. sNKG2DL have been shown to downmodulate NKG2D expression on the surface of $\mathrm{NK}$ cells and inhibit NK cell and T-cell functions in vitro [33-35]. Therefore, these data suggest that sNKG2DL may locally reduce recognition of tumor cells by diminishing NKG2DL densities at the cancer cell surface, but may also systematically impair NKG2D receptor function in cytotoxic immune effector cells. However, Zhang et al. showed that recombinant soluble MICA (at a concentration of as high as $1.5 \mu \mathrm{g} / \mathrm{ml}$ ) did not interfere with in vitro killing activity of NKR-2 T cells toward different tumor cells lines (K562 and RPMI8866). This suggests that sMICA in cancer patients may not inhibit the function of NKR-2 T cells in vivo [24]. Moreover, von Lilienfeld-Toal et al. showed that clinically relevant concentrations of sNKG2DL did not downregulate NKG2D expression in vitro [36]. Although we cannot exclude that levels of sNKG2DL at the tumor site could be different from the one measured in the serum, the fact that neither NKG2D expression nor NKG2D activity is affected by very high concentrations of sNKG2DL suggests that $s$ KKGDL should not interfere with NKR-2 T cell activity in vivo.

Taken together, direct antitumor activity, the potential effect on tumor neovasculature, the tumor microenvironment modulation, the putative insensitivity to sNKG2DL and the potential ability to target eight different ligands makes NKR-2 T cell a candidate that has the capacity to go beyond what is currently available for the 'standard' CAR T-cell approach.

\section{- Clinical development}

Since preclinical studies have shown the therapeutic potential of the murine chNKG2D T cells in both hematologic and solid tumor types and since the expression of NKG2DL was demonstrated on various solid cancer types, early clinical development programs started with CM-CS1 and THINK clinical trials. 
CM-CS1: initial clinical testing of NKR-2

A Phase I clinical study (CM-CS1) to establish the safety and feasibility of a single infusion of NKR-2 T cells without lymphodepletion started in April 2015 and was completed for enrollment in August 2016. Twelve patients with acute myeloid leukemia (AML)/myelodysplastic syndrome refractory anemia with excess blasts (MDS-RAEB), or relapsed/ refractory progressive MM without standard therapy options were infused with increasing doses of NKR-2 T cells (NCT02203825). The dose escalation involved four cohorts of patients starting at a low initial single dose of $10^{6}$ cells up to $3 \times 10^{7} \mathrm{NKR}-2$ cells per patient. The low doses reflect a prudent clinical development plan due to the novelty of the target. The initial clinical data presented a good safety profile since neither sign of cytokine release syndrome, nor tumor-lysis syndrome or off-tumor ontarget toxicity was observed. In addition, preclinical observations were corroborated since a short-term persistence of the NKR-2 cells in the peripheral circulation has also been observed. There were no objective responses by the day 28 evaluation. However, preliminary observations of unexpected survival and/or improvement in hematologic parameters were noted in both AML and MM patients with or without subsequent therapy as reported at the American Society of Hematology Annual meeting [37].

THINK: expanding clinical investigation of NKR-2 in solid \& hematological tumors The CM-CS1 clinical trial confirmed the initial safety and tolerability of a single dose of NKR-2 T cell. The target product profile of NKR-2 is derived from preclinical models involving three administrations of the NKR-2 product, 2 weeks apart $[20,22]$. Therefore, the THINK trial (EudraCT number 2016-00331212, NCT03018405) has been devised to test NKR-2 cell therapy further in the two hematological indications examined in CM-CS1 (MM, AML) and also in five solid tumor types (ovarian, colorectal, pancreas, bladder and triple-negative breast cancers). The THINK trial involves a dose escalation starting with three doses of $3 \times 10^{8}$ NKR-2 cells up to three doses of $3 \times 10^{9}$ cells, each dose being administered every 2 weeks. The primary objective of the THINK trial is safety and early signs of clinical efficacy. Data presented in this special report (Table 1 \& Figures 2-4) confirm that pancreatic tumor cells are targeted by human NKR-2 T cells in vitro and in vivo and support the ongoing solid tumor trial that opened to recruitment in January 2017 and is expected to be completed in mid-2018.

NKR-2 T cells display many potential advantages as compared with the 'standard' CAR T cell therapy. Multiple ligand targeting, lack of patient's preconditioning, induction of tumor specific adaptive immunity, anti-neoangiogenesis potential, activity even in NKG2DL heterogeneous tumors and modulation of the tumor microenvironment are combined to provide what could be an ideal therapeutic approach. However, preclinical mouse models rarely accurately predict responses in patients with advanced cancers. A specific concern is on-target/off-tumor activity and the multiple targeting capacity of stress-induced ligands by NKR-2. Pre-clinical models indeed suggest that very high doses of chNKG2D murine $\mathrm{T}$ cells infused as a bolus can induce toxicity in the mouse [38]. Similarly, preconditioning chemotherapies, combined with high doses of chNKG2D murine $\mathrm{T}$ cells, were shown to induce lethal toxicity in mice [39]. All these elements underscore the approach being taken in the Therapeutic Immunotherapy with NKR-2 (THINK) trial. The multiple dosing approach avoids reaching high doses where such toxicity is observed and allows a higher number of total cells to be given over a prolonged period.

\section{Future perspective}

In theory, the efficacy of a variety of different effector cells could be enhanced using the NKR-2 technology (CIK, NKT and NK cells) $[40-42]$. A group has published that NK cells transduced to express a chimeric NKG2D have a greater cytotoxicity [26]. Therefore, NKR-2 may also allow other effector cell types to recognize NKG2D ligands and makes them more effective against tumors. Whether NKR-2 T cells alone will be able to deliver a sufficient 'hit' against an established tumor in humans remains to be determined. However, the absence of preconditioning and the multiple dosing regimen currently used in the THINK trial provides an entirely suitable approach that allows combination with other therapies. One of the potential combinations would be with checkpoint inhibitors that have already shown some clinical benefits in solid tumors as a monotherapy [43]. The leverage of the immune brake by checkpoint inhibitors combined with 
NKR-2 could be a powerful solution to reach clinical efficacy. Alternatively, ionizing radiations are known to trigger NKG2DL expression on various cancer cell lines $[14,44]$, making the combination of this standard therapy with NKR-2 a potential association that might increase NKR-2 potency. Similarly, technologies enhancing NKR-2 T cells trafficking to tumors or modulating the tumor microenvironment (metabolically or immunologically) are all potential combinations for NKR2. How NKR-2 may be optimally combined with such therapies remains to be established. However, the multiple modes of action of NKR-2 provides a potential paradigm shift where CAR T cells and the induction of adaptive immunity together may become a game changer for the future of immuno-oncology.

Financial \& competing interests disclosure

$B$ Demoulin is an employee of Celyad SA. J Muradis is an employee of Celdara Medical. ML Sentman is related to a patent owner of this technology and is a consultant for Celdara Medical and Celyad. C Lonez is an employee of
Celyad, SA. D Gilhamis an employee of Celyad. CL Sentman is a Scientific Founder and consultant of Celdara Medical, LLC, holds patents on this technology and is a consultant for and has a sponsored research agreement with Celyad, SA. S Agaugue is an employee of Celyad $S A$. All this work was done under a Sponsored Research Agreement funded by Celyad SA. The authors have no other relevant affiliations or financial involvement with any organization or entity with a financial interest in or financial conflict with the subject matter or materials discussed in the manuscript apart from those disclosed.

No writing assistance was utilized in the production of this manuscript.

\section{Ethical conduct}

All animal work was performed at the Geisel School of Medicine Animal Facility in accordance with institutional guidelines.

Open access
This work is licensed under the Attribution-
NonCommercial-NoDerivatives 4.0 Unported License. To
view a copy of this license, visit http://creativecommons.org/
licenses/by-nc-nd/4.0/

\section{EXECUTIVE SUMMARY}

\section{NKR-2 chimeric antigen receptor T cells}

- NKR-2 chimeric antigen receptor (CAR) T cells express a chimeric construct created by fusing the full length NKG2D protein with the $\mathrm{CD} 3 \zeta$ intracellular domain. This construct is built as a first-generation CAR but act as a secondgeneration CAR due to DAP10 costimulatory signaling.

\section{Preclinical development}

- Murine chNKG2D T cells are efficient in vitro and in vivo against several solid and liquid tumors such as ovarian cancer, melanoma, lymphoma and multiple myeloma.

- New data presented in this special report demonstrate the in vitro antitumor efficacy of NKR-2 on colorectal and pancreatic cancer cell lines.

- In a model of xenograft of human pancreatic cancer, we demonstrate, for the first time, the in vivo antitumor efficacy of NKR-2 against an established pancreatic cancer.

- In addition to direct efficacy against a wide range of tumor cell lines or established tumors, preclinical data show that murine chNKG2D T cells:

Induce a long-term memory immune response against tumor antigen(s);

- Are effective without lymphodepletive preconditioning;

- Are effective against heterogeneous tumors;

- Impact tumor microenvironment by targeting immune-suppressive cells and neovasculature.

\section{Clinical development}

- The CM-CS1 clinical trial demonstrates the safety and feasibility of a low single-dose infusion of NKR-2 cells in hematological cancers.

- THINK trial expands clinical investigation made in CM-CS1 by testing multiple and higher doses. This trial is design to test NKR-2 in two hematological indications (acute myeloid leukemia and multiple myeloma) and in five solid cancers (ovarian, colorectal, pancreas, bladder and triple-negative breast cancers). 


\section{References}

Papers of special note have been highlighted as:

- of interest; $\bullet \bullet$ of considerable interest

1 Lee DW, Barrett DM, Mackall C, Orentas R, Grupp SA. The future is now: chimeric antigen receptors as new targeted therapies for childhood cancer. Clin. Cancer Res. 18(10), 2780-2790 (2012).

2 Dai H, Wang Y, Lu X, Han W. Chimeric antigen receptors modified T-cells for cancer therapy. J. Natl Cancer Inst. 108(7), pii: djv439 (2016).

3 Fousek K, Ahmed N. The evolution of T-cell therapies for solid malignancies. Clin. Cancer Res. 21(15), 3384-3392 (2015).

4 Yong CSM, Dardalhon V, Devaud C, Taylor N, Darcy PK, Kershaw MH. CAR T-cell therapy of solid tumors. Immunol. Cell Biol. 95(4), 356-363 (2017).

5 Newick K, O'Brien S, Moon E, Albelda SM. CAR T cell therapy for solid tumors. Annu. Rev. Med. 68, 139-152 (2017).

- Review article on chimeric antigen receptors (CAR) $\mathrm{T}$ cells outlining the advance and highlighting challenges that CAR T cell technology faces in solid tumor therapy.

6 Wang R, Jaw JJ, Stutzman NC, Zou Z, Sun PD. Natural killer cell-produced IFN- $\gamma$ and TNF- $a$ induce target cell cytolysis through up-regulation of ICAM-1. J. Leukoc. Biol. 91(2), 299-309 (2012).

7 Lanier LL. Up on the tightrope: natural killer cell activation and inhibition. Nat. Immunol. 9(5), 495-502 (2008).

8 Lanier LL. NKG2D receptor and its ligands in host defense. Cancer Immunol. Res. 3(6), 575-582 (2015).

- A complete review on the NKG2D receptor and its ligands. This review browses NKG2D role in host defense against infectious diseases and cancers and discusses its potential for therapy.

9 Coudert JD, Held W. The role of the NKG2D receptor for tumor immunity. Semin. Cancer Biol. 16(5), 333-343 (2006).

10 Diefenbach A, Tomasello E, Lucas M et al. Selective associations with signaling proteins determine stimulatory versus costimulatory activity of NKG2D. Nat. Immunol. 3(12), 1142-1149 (2002).

11 Zhang J, Basher F, Wu JD. NKG2D ligands in tumor immunity: two sides of a coin. Front. Immunol. 6, 97 (2015).

12 Raulet DH, Gasser S, Gowen BG, Deng W, Jung $H$. Regulation of ligands for the NKG2D activating receptor. Annu. Rev. Immunol. 31, 413-441 (2013).
13 Le Bert N, Gasser S. Advances in NKG2D ligand recognition and responses by NK cells. Immunol. Cell Biol. 92(3), 230-236 (2014).

14 Spear P, Wu M-R, Sentman M-L, Sentman CL. NKG2D ligands as therapeutic targets. Cancer Immun. 13, 8 (2013).

15 Sentman CL, Meehan KR. NKG2D CARs as cell therapy for cancer. Cancer J. Sudbury Mass. 20(2), 156-159 (2014).

- Review of the NKR-2 technology. This article reviews optimization made on NKR-2 and discusses potential toxicity associated.

16 Zhang T, Lemoi BA, Sentman CL. Chimeric NK-receptor-bearing $T$ cells mediate antitumor immunotherapy. Blood 106(5), 1544-1551 (2005).

-• The first article demonstrating that engineering murine $T$ cells with chimeric NKG2D receptor represents a promising approach for immunotherapy against cancer. This article shows chNKG2D efficiency on RMA cell line, a Rauscher's virus-induced lymphoma cell line.

17 Lanier LL, Corliss BC, Wu J, Leong C, Phillips JH. Immunoreceptor DAP12 bearing a tyrosine-based activation motif is involved in activating NK cells. Nature 391(6668), 703-707 (1998).

18 Wu J, Song Y, Bakker AB et al. An activating immunoreceptor complex formed by NKG2D and DAP10. Science 285(5428), 730-732 (1999).

19 Upshaw JL, Arneson LN, Schoon RA, Dick CJ, Billadeau DD, Leibson PJ. NKG2D-mediated signaling requires a DAP10-bound Grb2-Vav1 intermediate and phosphatidylinositol-3-kinase in human natural killer cells. Nat. Immunol. 7(5), 524-532 (2006).

20 Barber A, Zhang T, Sentman CL. Immunotherapy with chimeric NKG2D receptors leads to long-term tumor-free survival and development of host antitumor immunity in murine ovarian cancer. J. Immunol. 180(1), 72-78 (2008).

21 Zhang T, Sentman CL. Mouse tumor vasculature expresses NKG2D ligands and can be targeted by chimeric NKG2Dmodified T cells. J. Immunol. 190(5), 2455-2463 (2013).

22 Zhang T, Barber A, Sentman CL. Chimeric NKG2D modified T cells inhibit systemic T-cell lymphoma growth in a manner involving multiple cytokines and cytotoxic pathways. Cancer Res. 67(22), 11029-11036 (2007).

23 Barber A, Meehan KR, Sentman CL. Treatment of multiple myeloma with adoptively transferred chimeric NKG2D receptor-expressing T cells. Gene Ther. 18(5), 509-516 (2011).

24 Zhang T, Barber A, Sentman CL. Generation of antitumor responses by genetic modification of primary human $\mathrm{T}$ cells with a chimeric NKG2D receptor. Cancer Res. 66(11), 5927-5933 (2006).

- The first article demonstrating human NKR-2 efficacy against various cancer cell lines from multiple origins.

25 Barber A, Zhang T, DeMars LR, ConejoGarcia J, Roby KF, Sentman CL. Chimeric NKG2D receptor-bearing $T$ cells as immunotherapy for ovarian cancer. Cancer Res. 67(10), 5003-5008 (2007).

26 Chang Y-H, Connolly J, Shimasaki N, Mimura K, Kono K, Campana D. A chimeric receptor with NKG2D specificity enhances natural killer cell activation and killing of tumor cells. Cancer Res. 73(6), 1777-1786 (2013).

27 Spear P, Barber A, Rynda-Apple A, Sentman CL. NKG2D CAR T cell therapy inhibits the growth of NKG2D ligand heterogeneous tumors. Immunol. Cell Biol. 91(6), 435-440 (2013).

28 Almåsbak H, Aarvak T, Vemuri MC. CAR T cell therapy: a game changer in cancer treatment. J. Immunol. Res. 2016, 5474602 (2016).

29 Nausch N, Galani IE, Schlecker E, Cerwenka A. Mononuclear myeloid-derived "suppressor" cells express RAE-1 and activate natural killer cells. Blood 112(10), 4080-4089 (2008).

30 Roy S, Barnes PF, Garg A, Wu S, Cosman D, Vankayalapati R. NK cells lyse $T$ regulatory cells that expand in response to an intracellular pathogen. J. Immunol. 180 (3), 1729-1736 (2008).

31 Barber A, Rynda A, Sentman CL. Chimeric NKG2D expressing $T$ cells eliminate immunosuppression and activate immunity within the ovarian tumor microenvironment. J. Immunol. Baltim. Md 1950 183(11), 6939-6947 (2009).

32 Mou X, Zhou Y, Jiang P et al. The regulatory effect of UL-16 binding protein-3 expression on the cytotoxicity of NK cells in cancer patients. Sci. Rep. 4, 6138 (2014).

33 Song H, Kim J, Cosman D, Choi I. Soluble ULBP suppresses natural killer cell activity via down-regulating NKG2D expression. Cell. Immunol. 239(1), 22-30 (2006).

34 Groh V, Wu J, Yee C, Spies T. Tumourderived soluble MIC ligands impair expression of NKG2D and T-cell activation. Nature 419(6908), 734-738 (2002). 


\section{Exploiting natural killer group 2D Receptors for CAR T-cell therapy SPECIAL REPORT}

35 Nausch N, Cerwenka A. NKG2D ligands in tumor immunity. Oncogene 27(45), 5944-5958 (2008).

36 von Lilienfeld-Toal M, Frank S, Leyendecker $\mathrm{C}$ et al. Reduced immune effector cell NKG2D expression and increased levels of soluble NKG2D ligands in multiple myeloma may not be causally linked. Cancer Immunol. Immunother. 59(6), 829-839 (2010).

37 Nikiforow S, Murad J, Daley $\mathrm{H}$ et al. A first-in-human phase I trial of NKG2D chimeric antigen receptor-T cells in AML/ MDS and multiple myeloma. J. Clin. Oncol. 34(Suppl.), Abstract TPS3102 (2016).

38 Sentman M-L, Murad JM, Cook WJ et al. Mechanisms of acute toxicity in NKG2D chimeric antigen receptor $\mathrm{T}$ cell-treated mice. J. Immunol. 197(12), 4674-4685 (2016).

39 VanSeggelen H, Hammill JA, Dvorkin-Gheva A et al. $T$ cells engineered with chimeric antigen receptors targeting NKG2D ligands display lethal toxicity in mice. Mol. Ther. J. Am. Soc. Gene Ther. 23(10), 1600-1610 (2015).

40 Lu X, Zhu A, Cai X et al. Role of NKG2D in cytokine-induced killer cells against multiple myeloma cells. Cancer Biol. Ther. 13(8), 623-629 (2012).

41 Morisaki T, Hirano T, Koya $\mathrm{N}$ et al. NKG2D-directed cytokine-activated killer lymphocyte therapy combined with gemcitabine for patients with chemoresistant metastatic solid tumors. Anticancer Res. 34(8), 4529-4538 (2014).

42 Kuylenstierna C, Björkström NK, Andersson SK et al. NKG2D performs two functions in invariant NKT cells: direct TCR-independent activation of NK-like cytolysis and costimulation of activation by CD1d. Eur. J. Immunol. 41(7), 1913-1923 (2011).

43 Azoury SC, Straughan DM, Shukla V. Immune checkpoint inhibitors for cancer therapy: clinical efficacy and safety. Curr. Cancer Drug Targets 15(6), 452-462 (2015).

44 Kim J-Y, Son Y-O, Park S-W et al. Increase of NKG2D ligands and sensitivity to NK cell-mediated cytotoxicity of tumor cells by heat shock and ionizing radiation. Exp. Mol. Med. 38(5), 474-484 (2006).

45 Ge S-J, Duan L-N, Luo Y, Suo T-L, Lu C-R, Tang J. [Expression of NKG2D and NKG2A with their ligands MHC-I A/B and HLA-E in acute leukemia patients and its significance]. Zhongguo Shi Yan Xue Ye Xue Za Zhi 19(2), 312-316 (2011).

46 Hilpert J, Grosse-Hovest L, Grunebach F et al. Comprehensive analysis of NKG2D ligand expression and release in leukemia: implications for NKG2D-mediated NK cell responses. J. Immunol. 189(3), 1360-1371 (2012).

47 Jardine L, Hambleton S, Bigley V, Pagan S, Wang X-N, Collin M. Sensitizing primary acute lymphoblastic leukemia to natural killer cell recognition by induction of NKG2D ligands. Leuk. Lymphoma 54(1), 167-173 (2013).

48 Lanca T, Correia DV, Moita CF et al. The MHC class Ib protein ULBP1 is a nonredundant determinant of leukemia/ lymphoma susceptibility to T-cell cytotoxicity. Blood 115(12), 2407-2411 (2010).

49 Pende D. Analysis of the receptor-ligand interactions in the natural killer-mediated lysis of freshly isolated myeloid or lymphoblastic leukemias: evidence for the involvement of the poliovirus receptor (CD155) and Nectin-2 (CD112). Blood 105(5), 2066-2073 (2005).

50 Salih HR. Functional expression and release of ligands for the activating immunoreceptor NKG2D in leukemia. Blood 102(4), 1389-1396 (2003).

51 Satwani P, Bavishi S, Saha A et al. Upregulation of NKG2D ligands in acute lymphoblastic leukemia and non-Hodgkin lymphoma cells by romidepsin and enhanced in vitro and in vivo natural killer cell cytotoxicity. Cytotherapy 16(10), 1431-1440 (2014)

52 Baragaño Raneros A, Martín-Palanco V, Fernandez AF et al. Methylation of NKG2D ligands contributes to immune system evasion in acute myeloid leukemia. Genes Immun. 16(1), 71-82 (2015).

53 Diermayr S, Himmelreich H, Durovic B et al. NKG2D ligand expression in AML increases in response to HDAC inhibitor valproic acid and contributes to allorecognition by NK-cell lines with single KIR-HLA class I specificities. Blood 111(3), 1428-1436 (2008).

54 Epling-Burnette PK, Bai F, Painter JS et al. Reduced natural killer (NK) function associated with high-risk myelodysplastic syndrome (MDS) and reduced expression of activating NK receptors. Blood 109 (11), 4816-4824 (2007).

55 Farag SS, George SL, Lee EJ et al. Postremission therapy with low-dose interleukin 2 with or without intermediate pulse dose interleukin 2 therapy is well tolerated in elderly patients with acute myeloid leukemia: cancer and leukemia group B study 9420. Clin. Cancer Res. 8(9), 2812-2819 (2002).

56 Gundermann S, Klinker E, Kimmel B et al. A comprehensive analysis of primary acute myeloid leukemia identifies biomarkers predicting susceptibility to human allogeneic VY9V82 T cells. J. Immunother. 37(6), 321-330 (2014).

57 Khaznadar Z, Henry G, Setterblad N et al. Acute myeloid leukemia impairs natural killer cells through the formation of a deficient cytotoxic immunological synapse: innate immunity. Eur. J. Immunol. 44(10), 3068-3080 (2014).

58 Nanbakhsh A, Pochon C, Mallavialle A, Amsellem S, Bourhis JH, Chouaib S. c-Myc regulates expression of NKG2D ligands ULBP $1 / 2 / 3$ in AML and modulates their susceptibility to NK-mediated lysis. Blood 123(23), 3585-3595 (2014).

59 Nowbakht P, Ionescu M-CS, Rohner A et al. Ligands for natural killer cell-activating receptors are expressed upon the maturation of normal myelomonocytic cells but at low levels in acute myeloid leukemias. Blood 105(9), 3615-3622 (2005).

60 Poggi A, Catellani S, Garuti A, Pierri I, Gobbi M, Zocchi MR. Effective in vivo induction of NKG2D ligands in acute myeloid leukaemias by all-trans-retinoic acid or sodium valproate. Leukemia 23(4), 641-648 (2009).

61 Rohner A, Langenkamp U, Siegler U, Kalberer CP, Wodnar-Filipowicz A. Differentiation-promoting drugs up-regulate NKG2D ligand expression and enhance the susceptibility of acute myeloid leukemia cells to natural killer cell-mediated lysis. Leuk. Res. 31(10), 1393-1402 (2007).

62 Schlegel P, Ditthard K, Lang P et al. NKG2D signaling leads to nk cell mediated lysis of childhood AML. J. Immunol. Res. 2015, 1-10 (2015).

63 Cathro HP, Smolkin ME, Theodorescu D, Jo VY, Ferrone S, Frierson HF. Relationship between HLA class I antigen processing machinery component expression and the clinicopathologic characteristics of bladder carcinomas. Cancer Immunol. Immunother. CII 59(3), 465-472 (2010).

64 Orozco-Levi M, Ramírez-Sarmiento A, Borchers $\mathrm{M}$ et al. Lack of MICA expression predicts a worse prognosis in patients with bladder cancer. Open J. Pathol. 03(01), 41-50 (2013).

65 Crane CA, Han SJ, Barry JJ, Ahn BJ, Lanier LL, Parsa AT. TGF- downregulates the activating receptor NKG2D on NK cells and $\mathrm{CD}^{+} \mathrm{T}$ cells in glioma patients. Neuro Oncol. 12(1), 7-13 (2010).

66 Eisele G. TGF-beta and metalloproteinases differentially suppress NKG2D ligand surface expression on malignant glioma cells. Brain 129(9), 2416-2425 (2006). 
67 Friese MA, Platten M, Lutz SZ et al. MICA/ NKG2D-mediated immunogene therapy of experimental gliomas. Cancer Res. 63(24), 8996-9006 (2003).

68 Groh V, Rhinehart R, Secrist H, Bauer S, Grabstein KH, Spies T. Broad tumorassociated expression and recognition by tumor-derived gamma delta T cells of MICA and MICB. Proc. Natl Acad. Sci. USA 96(12), 6879-6884 (1999).

69 de Kruijf EM, Sajet A, van Nes JG et al. NKG2D ligand tumor expression and association with clinical outcome in early breast cancer patients: an observational study. BMC Cancer 12(1), 1 (2012).

70 Madjd Z, Spendlove I, Moss R et al. Upregulation of MICA on high-grade invasive operable breast carcinoma. Cancer Immun. Arch. 7(1), 17 (2007).

71 Mamessier E, Sylvain A, Thibult M-L et al. Human breast cancer cells enhance selftolerance by promoting evasion from NK cell antitumor immunity. J. Clin. Invest. 121(9), 3609-3622 (2011).

72 Mamessier E, Sylvain A, Bertucci F et al. Human breast tumor cells induce selftolerance mechanisms to avoid NKG2Dmediated and DNAM-mediated NK cell recognition. Cancer Res. 71(21), 6621-6632 (2011).

73 Arreygue-Garcia NA, Daneri-Navarro A, del Toro-Arreola A et al. Augmented serum level of major histocompatibility complex class I-related chain A (MICA) protein and reduced NKG2D expression on NK and $\mathrm{T}$ cells in patients with cervical cancer and precursor lesions. BMC Cancer 8(1), 16 (2008).

74 Textor S, Dürst M, Jansen L et al. Activating NK cell receptor ligands are differentially expressed during progression to cervical cancer. Int. J. Cancer 123(10), 2343-2353 (2008).

75 Nückel H, Switala M, Sellmann L et al. The prognostic significance of soluble NKG2D ligands in B-cell chronic lymphocytic leukemia. Leukemia 24(6), 1152-1159 (2010).

76 Poggi A, Venturino C, Catellani S et al. Vdelta1 T lymphocytes from B-CLL patients recognize ULBP3 expressed on leukemic B cells and up-regulated by trans-retinoic acid. Cancer Res. 64(24), 9172-9179 (2004).

77 Boissel N, Rea D, Tieng V et al. BCR/ABL oncogene directly controls MHC class I chain-related molecule a expression in chronic myelogenous leukemia. J. Immunol. 176(8), 5108-5116 (2006).

78 Sconocchia G, Spagnoli GC, Del Principe D et al. Defective infiltration of natural killer cells in MICA/B-positive renal cell carcinoma involves beta(2)-integrin-mediated interaction. Neoplasia NYN 11(7), 662-671 (2009).

79 McGilvray RW, Eagle RA, Watson NFS et al. NKG2D ligand expression in human colorectal cancer reveals associations with prognosis and evidence for immunoediting. Clin. Cancer Res. 15(22), 6993-7002 (2009).

80 Watson NFS, Spendlove I, Madjd Z et al. Expression of the stress-related MHC class I chain-related protein MICA is an indicator of good prognosis in colorectal cancer patients. Int. J. Cancer 118(6), 1445-1452 (2006).

81 Liu X, Sun M, Yu S, Liu K, Li X, Shi H. Potential therapeutic strategy for gastric cancer peritoneal metastasis by NKG2D ligands-specific T cells. OncoTargets Ther. 8, 3095-3104 (2015).

82 Mimura K, Kamiya T, Shiraishi K et al. Therapeutic potential of highly cytotoxic natural killer cells for gastric cancer: NK cytotoxicity against gastric cancer. Int. J. Cancer 135(6), 1390-1398 (2014).

83 Zhao S, Wang H, Nie Y, Mi Q, Chen X, Hou Y. Midkine upregulates MICA/B expression in human gastric cancer cells and decreases natural killer cell cytotoxicity. Cancer Immunol. Immunother. 61(10), 1745-1753 (2012).

84 Fang L, Gong J, Wang Y et al. MICA/B expression is inhibited by unfolded protein response and associated with poor prognosis in human hepatocellular carcinoma. J. Exp. Clin. Cancer Res. CR. 33, 76 (2014).

85 Jinushi M, Takehara T, Tatsumi $\mathrm{T}$ et al. Expression and role of MICA and MICB in human hepatocellular carcinomas and their regulation by retinoic acid. Int. J. Cancer 104(3), 354-361 (2003).

86 Jinushi M, Takehara T, Tatsumi $\mathrm{T}$ et al. Impairment of natural killer cell and dendritic cell functions by the soluble form of MHC class I-related chain A in advanced human hepatocellular carcinomas. J. Hepatol. 43(6), 1013-1020 (2005).

87 Kamimura H, Yamagiwa S, Tsuchiya A et al. Reduced NKG2D ligand expression in hepatocellular carcinoma correlates with early recurrence. J. Hepatol. 56(2), 381-388 (2012).

88 Baychelier F, Achour A, Nguyen S et al. Natural killer cell deficiency in patients with non-Hodgkin lymphoma after lung transplantation. J. Heart Lung Transplant. 34(4), 604-612 (2015).

89 Camodeca C, Nuti E, Tepshi L et al. Discovery of a new selective inhibitor of A
Disintegrin And Metalloprotease 10 (ADAM-10) able to reduce the shedding of NKG2D ligands in Hodgkin's lymphoma cell models. Eur. J. Med. Chem. 111, 193-201 (2016).

90 Capriotti E, Vonderheid EC, Thoburn CJ, Wasik MA, Bahler DW, Hess AD. Expression of T-plastin, FoxP3 and other tumorassociated markers by leukemic T-cells of cutaneous T-cell lymphoma. Leuk. Lymphoma 49(6), 1190-1201 (2008).

91 Cox ST, Pearson H, Laza-Briviesca R et al. Characterization of $5^{\prime}$ promoter and exon 1-3 polymorphism of the RAET1E gene. Hum. Immunol. 77(1), 96-103 (2015).

92 Dulphy N, Berrou J, Campillo JA, Bagot M, Bensussan A, Toubert A. NKG2D ligands expression and NKG2D-mediated NK activity in Sezary patients. J. Invest. Dermatol. 129(2), 359-364 (2009).

93 Kim JH, Lee JK. Naringenin enhances NK cell lysis activity by increasing the expression of NKG2D ligands on Burkitt's lymphoma cells. Arch. Pharm. Res. 38(11), 2042-2048 (2015).

94 Kim JH, Lee JK. Sesamolin enhances NK cell lysis activity by increasing the expression of NKG2D ligands on Burkitt's lymphoma cells. Int. Immunopharmacol. 28(2), 977-984 (2015).

95 Zocchi MR, Catellani S, Canevali P et al. High ERp5/ADAM10 expression in lymph node microenvironment and impaired NKG2D ligands recognition in Hodgkin lymphomas. Blood 119(6), 1479-1489 (2012).

96 Maccalli C, Nonaka D, Piris A et al. NKG2D-mediated antitumor activity by tumor-infiltrating lymphocytes and antigen-specific T-cell clones isolated from melanoma patients. Clin. Cancer Res. 13(24), 7459-7468 (2007).

97 Paschen A, Sucker A, Hill B et al. Differential clinical significance of individual NKG2D ligands in melanoma: soluble ULBP2 as an indicator of poor prognosis superior to S100B. Clin. Cancer Res. 15(16), 5208-5215 (2009).

98 Vetter CS, Groh V, thor Straten P, Spies T, Bröcker E-B, Becker JC. Expression of stress-induced MHC class I related chain molecules on human melanoma. J. Invest. Dermatol. 118(4), 600-605 (2002).

99 Vetter CS, Lieb W, Bröcker E-B, Becker JC. Loss of nonclassical MHC molecules MIC-A/B expression during progression of uveal melanoma. Br. J. Cancer 91(8), 1495-1499 (2004).

100 Carbone E. HLA class I, NKG2D, and natural cytotoxicity receptors regulate 
multiple myeloma cell recognition by natural killer cells. Blood 105(1), 251-258 (2005).

101 Girlanda S, Fortis C, Belloni D et al. MICA expressed by multiple myeloma and monoclonal gammopathy of undetermined significance plasma cells costimulates pamidronate-activated gammadelta lymphocytes. Cancer Res. 65(16), 7502-7508 (2005).

102 Jinushi M, Vanneman M, Munshi NC et al. MHC class I chain-related protein A antibodies and shedding are associated with the progression of multiple myeloma. Proc. Natl Acad. Sci. USA 105(4), 1285-1290 (2008).

103 Meehan KR, Talebian L, Tosteson TD et al. Adoptive cellular therapy using cells enriched for $\mathrm{NKG} 2 \mathrm{D}^{+} \mathrm{CD} 3{ }^{+} \mathrm{CD} 8{ }^{+} \mathrm{T}$ cells after autologous transplantation for myeloma. Biol. Blood Marrow Transplant. 19(1), 129-137 (2013).

104 Okamoto M, Inaba T, Yamada N et al. Expression and role of MHC class I-related chain in myeloma cells. Cytotherapy 8(5), 509-516 (2006).

105 von Strandmann EP, Hansen HP, Reiners KS et al. A novel bispecific protein (ULBP2-BB4) targeting the NKG2D receptor on natural killer (NK) cells and CD138 activates NK cells and has potent antitumor activity against human multiple myeloma in vitro and in vivo. Blood 107(5), 1955-1962 (2006).

106 Raffaghello L, Prigione I, Airoldi I et al. Downregulation and/or release of NKG2D ligands as immune evasion strategy of human neuroblastoma. Neoplasia 6(5), 558-568 (2004).

107 Busche A, Goldmann T, Naumann U, Steinle A, Brandau S. Natural killer cell-mediated rejection of experimental human lung cancer by genetic overexpression of major histocompatibility complex class I chainrelated gene A. Hum. Gene Ther. 17(2), 135-146 (2006).

108 Chen Y, Lin G, Guo Z, Zhou Z, He Z, Ye Y. Effects of MICA expression on the prognosis of advanced non-small cell lung cancer and the efficacy of CIK therapy. PLoS ONE 8(7), e69044 (2013).

109 Platonova S, Cherfils-Vicini J, Damotte D et al. Profound coordinated alterations of intratumoral NK cell phenotype and function in lung carcinoma. Cancer Res. 71(16), 5412-5422 (2011).

110 Barber A, Zhang T, DeMars LR, ConejoGarcia J, Roby KF, Sentman CL. Chimeric NKG2D receptor-bearing T cells as immunotherapy for ovarian cancer. Cancer Res. 67(10), 5003-5008 (2007).

111 Conejo-Garcia JR, Benencia F, Courreges MC et al. Ovarian carcinoma expresses the NKG2D ligand Letal and promotes the survival and expansion of CD28- antitumor T cells. Cancer Res. 64(6), 2175-2182 (2004).

112 Li K, Mandai M, Hamanishi J et al. Clinical significance of the NKG2D ligands, MICA/B and ULBP2 in ovarian cancer: high expression of ULBP2 is an indicator of poor prognosis. Cancer Immunol. Immunother. 58(5), 641-652 (2009).

113 McGilvray RW, Eagle RA, Rolland P, Jafferji I, Trowsdale J, Durrant LG. ULBP2 and RAET1E NKG2D ligands are independent predictors of poor prognosis in ovarian cancer patients. Int. J. Cancer 127(6), 1412-1420 (2010).

114 Duan X, Deng L, Chen X et al. Clinical significance of the immunostimulatory MHC class I chain-related molecule A and NKG2D receptor on NK cells in pancreatic cancer. Med. Oncol. 28(2), 466-474 (2011).

115 Gong W, Xiao W, Gong C, Tian F, Ji M. [Association of MICA gene polymorphism and serum soluble MICA level with colorectal cancer]. Zhonghua Yi Xue Yi Chuan Xue Za Zhi 27(3), 335-339 (2010).

116 Xu X, Rao GS, Groh V et al. Major histocompatibility complex class I-related chain A/B (MICA/B) expression in tumor tissue and serum of pancreatic cancer: role of uric acid accumulation in gemcitabineinduced MICA/B expression. BMC Cancer 11(1), 194 (2011).

117 Wu JD, Higgins LM, Steinle A, Cosman D, Haugk K, Plymate SR. Prevalent expression of the immunostimulatory MHC class I chain-related molecule is counteracted by shedding in prostate cancer. J. Clin. Invest. 114(4), 560-568 (2004).

118 Viey E, Fromont G, Escudier B et al. Phosphostim-activated T cells kill autologous metastatic renal cell carcinoma. J. Immunol. 174(3), 1338-1347 (2005).

119 Zhang X, Yan L, Jiao W et al. The clinical and biological significance of MICA in clear cell renal cell carcinoma patients. Tumour Biol. 37(2), 2153-2159 (2015).

120 Berghuis D, Schilham MW, Vos HI et al. Histone deacetylase inhibitors enhance expression of NKG2D ligands in Ewing sarcoma and sensitize for natural killer cell-mediated cytolysis. Clin. Sarcoma Res. 2(1), 8 (2012).

121 Verhoeven DHJ, de Hooge ASK, Mooiman ECK et al. NK cells recognize and lyse Ewing sarcoma cells through NKG2D and DNAM-1 receptor dependent pathways. Mol. Immunol. 45(15), 3917-3925 (2008). 